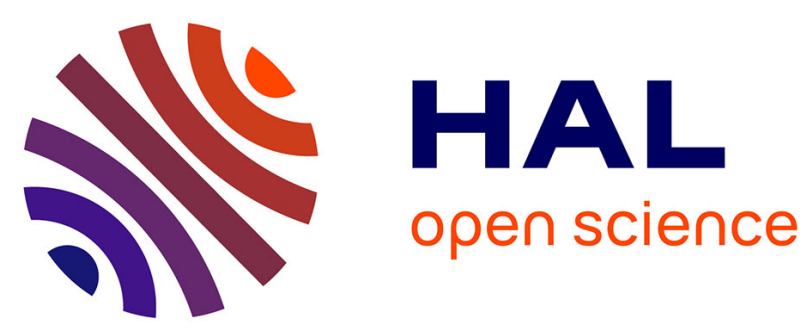

\title{
CAPPEAU PAUL \& ROUBAUD MARIE-NOËLLE. REGARDS LINGUISTIQUES SUR LES TEXTES D'ÉLÈVES (DE 5 À 12 ANS)
}

Lionel Audion

\section{> To cite this version:}

Lionel Audion. CAPPEAU PAUL \& ROUBAUD MARIE-NOËLLE. REGARDS LINGUISTIQUES SUR LES TEXTES D'ÉLÈVES (DE 5 À 12 ANS). Revue Française de Pédagogie, 2018. hal-02459579

\section{HAL Id: hal-02459579 \\ https://hal.science/hal-02459579}

Submitted on 29 Jan 2020

HAL is a multi-disciplinary open access archive for the deposit and dissemination of scientific research documents, whether they are published or not. The documents may come from teaching and research institutions in France or abroad, or from public or private research centers.
L'archive ouverte pluridisciplinaire HAL, est destinée au dépôt et à la diffusion de documents scientifiques de niveau recherche, publiés ou non, émanant des établissements d'enseignement et de recherche français ou étrangers, des laboratoires publics ou privés. 
CAPPEAU PAUL \& ROUBAUD MARIE-NOËLLE. REGARDS LINGUISTIQUES SUR LES TEXTES D'ÉLĖVES (DE 5 À 12 ANS)

Clermont-Ferrand : Presses universitaires Blaise-Pascal, 2018, 372 p.

\section{Lionel Audion}

E.N.S. Editions | « Revue française de pédagogie »

2018/1 n² 202 | pages 139 à 141

ISSN 0556-7807

Article disponible en ligne à l'adresse :

https://www.cairn.inforevue-francaise-de-pedagogie-2018-1-page-139.htm

Distribution électronique Cairn.info pour E.N.S. Editions.

(C) E.N.S. Editions. Tous droits réservés pour tous pays.

La reproduction ou représentation de cet article, notamment par photocopie, n'est autorisée que dans les limites des conditions générales d'utilisation du site ou, le cas échéant, des conditions générales de la licence souscrite par votre établissement. Toute autre reproduction ou représentation, en tout ou partie, sous quelque forme et de quelque manière que ce soit, est interdite sauf accord préalable et écrit de l'éditeur, en dehors des cas prévus par la législation en vigueur en France. Il est précisé que son stockage dans une base de données est également interdit. 
山्山FARAÇAISE

$\gg \mathrm{DE}$

핌AGOGIE

\section{Revue française de pédagogie}

Recherches en éducation

$202 \mid 2018$

Recherche, politique et pratiques en éducation :

services rendus et questions posées d'un univers à

l'autre /3

\section{CAPPEAU Paul \& ROUBAUD Marie-Noëlle. Regards linguistiques sur les textes d'élèves (de 5 à 12 ans)}

Clermont-Ferrand : Presses universitaires Blaise-Pascal, 2018, 372 p.

\section{Lionel Audion}

\section{Q OpenEdition \\ Journals}

Édition électronique

URL : http://journals.openedition.org/rfp/7589

ISSN : 2105-2913

Éditeur

ENS Éditions

Édition imprimée

Date de publication : 31 mars 2018

Pagination : 139-141

ISBN : 979-10-362-0184-4

ISSN : 0556-7807

Distribution électronique Cairn

\section{CAIRN, INFO}

CHERCHER, REPÉRER, AVANCER.

\section{Référence électronique}

Lionel Audion, «CAPPEAU Paul \& ROUBAUD Marie-Noëlle. Regards linguistiques sur les textes d'élèves (de 5 à 12 ans)», Revue française de pédagogie [En ligne], 202 | 2018, mis en ligne le 31 mars 2018, consulté le 12 septembre 2019. URL : http://journals.openedition.org/rfp/7589

Ce document a été généré automatiquement le 12 septembre 2019.

(c) tous droits réservés 


\title{
CAPPEAU Paul \& ROUBAUD Marie- Noëlle. Regards linguistiques sur les textes d'élèves (de 5 à 12 ans)
}

Clermont-Ferrand: Presses universitaires Blaise-Pascal, 2018, 372 p.

\author{
Lionel Audion
}

\section{RÉFÉRENCE}

CAPPEAU Paul \& ROUBAUD Marie-Noëlle. Regards linguistiques sur les textes d'élèves (de $5 \grave{a}$ 12 ans). Clermont-Ferrand : Presses universitaires Blaise-Pascal, 2018, 372 p.

1 Cet ouvrage sera très précieux aux linguistes qui souhaitent travailler sur des corpus de productions écrites d'enfants ; il sera également un outil utile pour préparer les concours de recrutement, notamment le CRPE (Concours de recrutement de professeur des écoles), selon une démarche scientifique solide. Son originalité et son principal atout viennent de l'entrée choisie par les auteurs, qui ont compilé près de cent productions écrites d'élèves, du $\mathrm{CP}$ au $\mathrm{CM} 2$ et un peu dans les premières années du collège. Cette démarche était déjà au cœur de leur précédent ouvrage (Cappeau \& Roubaud, 2005), mais cette fois l'ambition est plus grande car il s'agit également d'une somme de connaissances linguistiques mises à disposition des lecteurs : on n'y trouve pas de parti pris théorique global (ce qui déroute parfois, comme dans le chapitre 10 sur le lexique: (f. infra); pour chaque chapitre, Cappeau et Roubaud sélectionnent des théories, en une sorte d'état de l'art qui sera également utile aux étudiants. L'ensemble est néanmoins très saussurien, et il est agréable de constater que quelques linguistes un peu oubliés du début du $\mathrm{XX}^{\mathrm{e}}$ siècle sont parfois convoqués, comme Joseph Vendryes et Antoine Meillet ; les auteurs n'hésitent pas non plus à se référer incidemment à des théories considérées comme ardues: Gustave Guillaume, ainsi que Damourette et Pichon. Mais pour l'ensemble, ils vont aux valeurs sûres : on ne sera pas étonné de retrouver Chervel, Catach, Cogis, Fayol et Jaffré dans le chapitre consacré aux erreurs d'orthographe par exemple. 
2 Une autre ligne directrice des auteurs est celle d'insister sur les rapports entre l'oral et l'écrit, en citant fréquemment les travaux de Blanche-Benveniste et en n'hésitant pas à mettre en avant parfois une grammaire de l'oral, particulièrement intéressante par exemple dans le chapitre 5 sur le genre et le nombre; il est important que les lecteurs prennent conscience de la prégnance de l'oral sur les productions écrites des jeunes élèves.

3 Signalons également la très bonne préface de Claudine Garcia-Debanc, qui voit dans cette cartographie des erreurs d'élèves l'esquisse d'une " grammaire de la production écrite (qui requiert d'autres entrées qu'une grammaire de réception)» (p. 11).

4 Les onze chapitres de ces « regards linguistiques sur les textes d'élèves » sont construits avec grande rigueur (chacun correspondant à un problème d'écriture), selon un modèle unique et très facilitateur : un bref échantillon de productions d'élèves ouvre le chapitre et pose le problème ; viennent ensuite des extraits de textes officiels qui permettent au lecteur de reconstituer l'historique de la façon dont les notions ont été traitées dans les programmes d'enseignement, depuis la «maîtrise de la langue » en 1992, jusqu'aux programmes de 2015. On regrettera toutefois que les tout derniers décrets et mises à jour des programmes n'aient pas pu être pris en compte, puisque l'approche de l'étude de la langue à l'école a été grandement recadrée en 2018. Viennent ensuite des "clés pour comprendre »: il s'agit de véritables condensés de cours qui permettent de cerner le problème et d'analyser les textes d'élèves, à la lumière des théories linguistiques. La seconde partie de chaque chapitre est consacrée à des analyses en profondeurs de productions d'élèves, très éclairantes, et se nourrissant au fur et à mesure des apports des chapitres précédents. Enfin, des propositions bibliographiques (ouvrages ou articles), volontairement réduites et parfois commentées, terminent les chapitres.

5 Les deux premiers chapitres («Écrire les sons » et «Les découpages du texte») sont consacrés aux problèmes rencontrés par les élèves lors de l'entrée dans l'écrit. Après un rappel utile sur la différence entre phonétique et phonologie, les auteurs analysent des erreurs récurrentes touchant à la phase de codage. Le chapitre 2 propose d'intéressants outils pour classifier les erreurs de sous ou sur-segmentation (p. 71 et 73). Notons que les auteurs n'hésitent pas à donner leur avis sur les programmes : «Les programmes de 2008 proposent une progression (du simple au complexe) qui peut susciter des questions et des réserves. La hiérarchie des unités proposées a en effet de quoi surprendre » (p. 53).

6 Les deux chapitres suivants sont consacrés à la gestion des personnages (leur désignation et les paroles rapportées). Le chapitre 3 insiste sur la notion de cohésion (celle de cohérence sera abordée à la fin du volume). Le principal intérêt du chapitre 4 est d'instaurer les notions utiles de « discours citant » et « discours cité » (p. 116). Les auteurs rappellent également que «l'indépendance grammaticale entre ces deux parties du discours ne doit pas faire illusion: un seul énonciateur est effectivement impliqué » (p. 117) en analysant des « discours citant » tels que « prétendit-il ».

7 Le chapitre 5 s'attache aux variations qui touchent le nom et l'adjectif. Chapitre capital, remarquablement bien construit, qui rappelle aux lecteurs le rôle de la langue orale dans les productions écrites des élèves. Pour le féminin par exemple, les auteurs rappellent que "l'école tend à établir un lien étroit entre la notion de féminin et la marque graphique “e". Cette règle n'est vraie, à l'écrit, que pour un nombre limité d'adjectifs ("jolie", "forte", “petite") ; elle n'est jamais valable à l'oral ([gri], [griz])» (p. 150). C'est aussi à ce chapitre que Gustave Guillaume est convoqué à propos de la distinction précieuse entre genre vrai 
et genre arbitraire. Les analyses de textes d'élèves portant sur le pluriel sont également très pertinentes, en particulier autour des personnes du pluriel en conjugaison, ce qui fait la transition avec les chapitres suivants.

8 Les chapitres 6 et 7 sont consacrés au verbe. À part la comparaison de la morphologie verbale à l'écrit et à l'oral (inspirée de Blanche-Benveniste) et la réflexion sur les bases, cette partie, très classique, est un peu décevante, car elle ne prend pas en compte les recherches récentes en didactique du verbe, par exemple celles de Patrice Gourdet (2016). C'est encore plus vrai pour le chapitre 7 , entièrement écrit sous l'éclairage des deux systèmes d'énonciation d'Émile Benveniste; des textes d'élèves offraient pourtant l'occasion de traiter de l'usage fréquent du passé composé dans les récits d'élèves, en système avec le passé simple, mais ce problème est à peine survolé.

9 Les trois chapitres suivants sont consacrés à l'orthographe, la grammaire et le vocabulaire. Le chapitre 8 commence par un intéressant rappel historique des débats sur l'orthographe depuis Meigret (1550) ; les auteurs comparent ensuite l'analyse d'erreurs selon Catach puis selon Chervel et Blanche-Benveniste, ce qui est très pertinent et permet aux chercheurs (ou candidats aux concours de l'enseignement) de sélectionner la démarche qui convient le mieux selon le texte à étudier, comme les exemples proposés dans le chapitre le montrent au travers d'analyses très riches, qui mettent en évidence le développement progressif de la conscience orthographique chez les élèves.

Le chapitre 9 permettra aux lecteurs de délimiter très clairement ce qu'est une « erreur de grammaire » (c'est-à-dire de syntaxe). Les auteurs insistent sur trois types d'erreurs très répandus : la construction des verbes, les constructions suspendues et les problèmes avec la proposition relative. Pour finir, ils combattent à juste titre l'idée que ces erreurs, à l'écrit, viennent de l'influence de la langue orale, a priori qu'ils attribuent à une « vision obsolète et erronée de la langue orale » (p. 282).

11 Le chapitre 10 quant à lui permet de rappeler la différence entre lexique (ensemble des mots $d u$ français) et vocabulaire (portion du lexique habituellement employée par tel locuteur) selon Picoche ; suit un rappel théorique sur les étapes de l'acquisition des mots chez le jeune enfant; les auteurs empruntent d'abord, pour ce qui concerne le nom, à Bassano (2010) connue pour sa théorie de la masse lexicale critique, qui s'inscrit dans la famille théorique des modèles de compétition, puis vont chercher David (2000) pour les formes non nominales, ce qui peut étonner puisqu'il aurait été plus cohérent de continuer avec Bassano, laquelle a également produit une théorie de l'acquisition du verbe (2010). Les auteurs rappellent ensuite opportunément l'organisation du lexique selon Saussure (en discours et en langue), qu'ils prolongent par les apports de Colin (2003) sur les orientations sémasiologique ( $\mathrm{du}$ mot vers le sens) et onomasiologique (du concept vers ses expressions en langue). L'idée force de ce chapitre est qu'«au-delà de l'emploi ordinaire du mot, ce sont les organisations du lexique qui intéressent [les auteurs]» (p. 314). Les analyses de textes d'élèves qui suivent illustrent très bien ces rappels théoriques, notamment la démarche onomasiologique adoptée dans le texte « la dispute » (p. 321).

12 Le chapitre 11 porte sur la cohérence, « ce qui permet de passer d'un ensemble de phrases à un texte » (p.327), notion que les auteurs préfèrent appeler «textualité ». Ce dernier chapitre couronne logiquement cet ouvrage dont le fil conducteur a été cette volonté de dépasser le mot, la phrase, pour concevoir les productions d'élèves comme un tout, et donc analyser les erreurs à l'échelle du texte. Ce souci permet entre autres à Cappeau et Roubaud de toujours « faire ressortir les nombreux points positifs auxquels un regard non 
expert n'est pas toujours sensible» (p. 327). Il est intéressant à ce sujet de comparer les analyses du texte de Charlotte, CE2, cohérent car conforme à l'univers du conte, à celui de Lilia, qui ne parvient pas à maintenir sa position énonciative.

13 Nous dirons pour conclure que ce livre est précieux pour entrer dans les théories linguistiques au moyen d'un corpus riche qui redonne toute sa noblesse à ce genre particulier qu'est le texte d'élève. Il sera également utile aux candidats au CRPE. L'ouvrage est roboratif et nourri de 167 références bibliographiques. On pourra toutefois regretter de ne compter que 17 références postérieures à 2010, dont 5 sont des autoréférences des deux auteurs.

\section{BIBLIOGRAPHIE}

BASSANO D. (2010). «L'acquisition des verbes en français : un exemple de l'interface lexique/ grammaire ». Synergies France, $n^{\circ}$ 6, p. 27-39.

CAPPEAU P. \& ROUBAUD M.-N. (2005). Enseigner les outils de la langue avec des productions d'élèves. Paris : Bordas.

COLIN J.-P. (2003). « Le lexique ». In M. Yaguello (dir.), Le grand livre de la langue française. Paris : Éd. du Seuil, p. 391-456.

DAVID J. (2000). « Le lexique et son acquisition : aspects cognitifs et linguistiques ». Le français aujourd'hui, nº 131, p. 31-41.

GOURDET P. (2016). « Le verbe au sein des manuels à l'école primaire : quelles articulations entre savoir scolaire et savoir linguistique ? ». Français Aujourd'hui, n 194, p. 47-57.

\section{AUTEURS}

\section{LIONEL AUDION}

Université de Nantes, CREN (EA 2661) 